\title{
Recubrimientos decorativos semitransparentes realizados mediante pulverización catódica
}

\author{
Enrique Carretero, Rafael Alonso \\ Grupo de Tecnologías Fotónicas (GTF) \\ Instituto de Investigación en Ingeniería de Aragón (I3A) \\ Universidad de Zaragoza, Mariano Esquillor s/n, 50018, Zaragoza, Spain. \\ Tel. +34-976762707, e-mail: ecarre@unizar.es
}

\begin{abstract}
El presente trabajo aborda el desarrollo de recubrimientos decorativos con propiedades ópticas controladas. Se presenta una propuesta de multicapa así como un análisis de las posibilidades estéticas que ofrece.
\end{abstract}

\section{Introducción}

Las curvas espectrales de reflexión y transmisión de recubrimientos de capas finas formadas por múltiples capas pueden ajustarse haciendo uso de la interferencia entre las múltiples reflexiones en las intercaras formadas entre las capas [1]. Este fenómeno es ampliamente usado para la fabricación de filtros ópticos.

\section{Material y métodos}

Los recubrimientos fueron depositados mediante la técnica de pulverización catódica asistida por campo magnético o "magnetron sputtering" en una instalación a escala piloto industrial capaz de realizar recubrimientos homogéneos sobre grandes áreas planas de hasta 600x300mm.

Como materiales, se usaron capas metálicas de Sst 316 y capas dieléctricas de SiAlNx haciendo uso de sputtering reactivo a partir de un blanco de una aleación de Si (90\%) y Al (10\%) [2]. El tamaño de los blancos de material fue de 600x100mm.

Las deposiciones han sido realizadas en un vacio con una presión base del orden de $10^{-6} \mathrm{mbar}$ e introduciendo los gases de proceso, generalmente Ar en sputtering no reactivo y $\mathrm{N}_{2}$ en caso de realizar sputtering reactivo, hasta alcanzar una presión de trabajo del orden de $10^{-3} \mathrm{mbar}$, lo que equivale a un flujo total de aproximadamente 200sccm para la instalación utilizada. Para la pulverización de los blancos se utilizó una potencia de $1 \mathrm{~kW}$ para el Sst 316 y un flujo de 200sccm de Ar mientras que en el caso del SiAl se usó una potencia de 2,5kW y unos flujos de 100 sccm de $\mathrm{N}_{2}$ y 100 sccm de Ar.
La caracterización óptica fue realizada con un espectrofotómetro capaz de medir reflexión por ambas caras y transmisión del recubrimiento en un rango de longitudes de onda desde 300 a 2500nm. De esta forma, pueden calcularse las coordenadas de color CIELAB 1976, así como la diferencia de color entre dos muestras como

$$
\Delta E=\sqrt{(\Delta L)^{2}+(\Delta a)^{2}+(\Delta b)^{2}}
$$

La estructura multicapa finalmente estudiada, estuvo formada por un total de 5 capas del tipo: Sustrato/Dieléctrico/Metal/Dieléctrico/Metal/Dielec trico [3].

\section{Resultados y discusión}

La estructura multicapa con 5 capas ha demostrado una amplia flexibilidad para seleccionar el aspecto estético del recubrimiento con gran independencia de la transmición óptica requerida, debido a que la reflexión del recubrimiento viene especialmente definida por las 3 primeras capas del recubrimiento. La cuarta capa, será un metal con gran absorción óptica, de forma que mediante el control de su espesor puede determinarse la transmisión óptica que tendrá el recubrimiento. Por último, la quinta capa únicamente tendrá funciones de protección, debido a su mayor resistencia mecánica y la labor de barrera que hace para evitar que las capas metálicas interiores se oxiden.

Se han realizado recubrimientos de diversas tonalidades, Figura 1, siendo la estructura en todos casos similar, cambiando únicamente el espesor de las capas.

Así mismo, se hicieron muestras que teniendo una diferencia de color con $\Delta E<3$ en reflexión, tuvieran diferentes transmitancias, Tabla 1 , esto se consiguió modificando especialmente el espesor de la cuarta capa, encargada de determinar la opacidad del recubrimientos, modificando el espesor de esta capa entre 20 y $35 \mathrm{~nm}$ se pueden obtener 
transmitancias que varían entre el 14 y el 5\%, Figura 2, mientras que en el resto de capas apenas hubo que hacer ligeros ajustes.

Tabla 1: Estructura de capas que forman el recubrimiento y sus características ópticas de transmisión y reflexión.

\begin{tabular}{|c|c|c|c|}
\hline Capa no & Material & $\mathbf{d}_{\mathbf{1}}(\mathbf{n m})$ & $\mathbf{d}_{\mathbf{2}}(\mathbf{n m})$ \\
\hline $\mathbf{1}$ & SiAIN $_{\mathrm{x}}$ & 16 & 16 \\
\hline $\mathbf{2}$ & Sst & 8 & 8 \\
\hline $\mathbf{3}$ & SiAIN $_{\mathrm{x}}$ & 86 & 82 \\
\hline $\mathbf{4}$ & Sst & 20 & 35 \\
\hline $\mathbf{5}$ & SiAIN $_{\mathrm{x}}$ & 50 & 50 \\
\hline \hline \multicolumn{2}{|c|}{ Transmisión (\%) } & 14 & 5 \\
\hline \hline \multirow{2}{*}{$\begin{array}{c}\text { Coordenadas } \\
\text { de color } \\
\text { (reflexión) }\end{array}$} & $\mathrm{L}$ & 53,6 & 55,3 \\
\cline { 2 - 4 } & $\mathrm{a}$ & $-7,5$ & $-8,9$ \\
\hline \multicolumn{2}{|c}{$\mathrm{b}$} & $-25,3$ & $-25,4$ \\
\hline
\end{tabular}

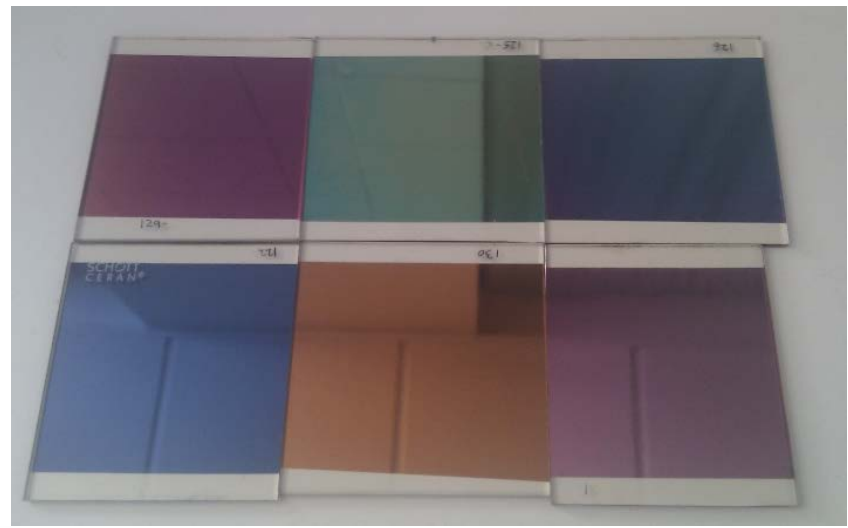

Figura 1: Muestras realizadas de diferentes tonalidades en reflexión.

\section{Conclusiones}

La deposición de recubrimientos decorativos semitransparentes mediante la técnica de sputtering ha demostrado tener una amplia flexibilidad en cuanto a la posibilidad de escoger tanto el aspecto estético en reflexión como el poder seleccionar el valor de la transmisión óptica simultáneamente. Se ha comprobado como una estructura adecuada puede adaptarse a los requerimientos deseados con pequeñas modificaciones.

\section{REFERENCIAS}

[1]. THELEN, A. Design of Optical Interference Coatings. New York: McGraw-Hill, 1989.

[2]. MA, P., LIN, F., DOBROWOLSKY, J.A. Design and manufacture of metal/dielectric long-wavelength cutoff filters. Applied Optics. 2001, 50(9), C201-C209.

[3]. BSH ELECTRODOMESTICOS ESPAÑA. Placa de cocción, y campo de cocción con una placa de campo de cocción correspondiente. España, Patente No 2392713. 04Oct-2012.

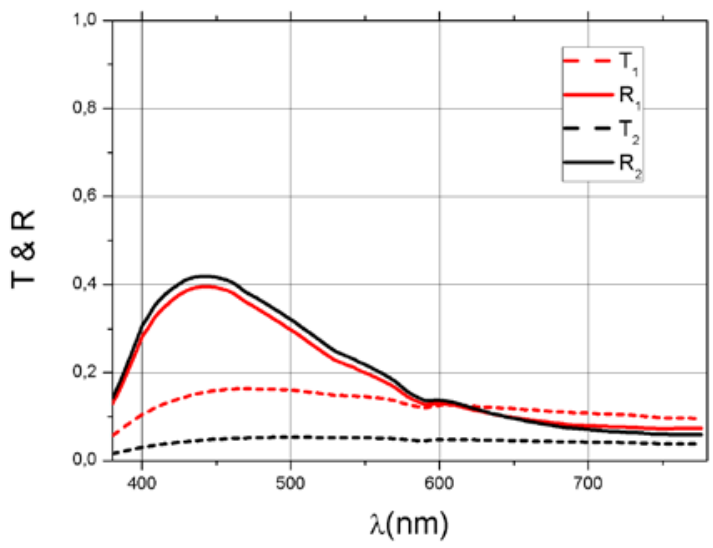

Figura 2: Comparación de espectros de transmisión y reflexión de diferentes muestras. 